\title{
Left Ventricular Function by Pressure-Volume Loop Analysis before and after Percutaneous Repair of Large Atrial Septal Defects
}

\author{
D. S. LIM, M.D., ${ }^{1}$ HOWARD P. GUTGESELL, M.D., ${ }^{2}$ and ALBERT P. ROCCHINI, M.D. ${ }^{3}$ \\ From the ${ }^{1}$ Assistant Professor of Pediatrics \& Medicine, University of Virginia, Charlottesville, Virginia; ${ }^{2}$ Professor of Pediatrics, University \\ of Virginia, Charlottesville, Virginia; and ${ }^{3}$ Professor of Pediatrics, University of Michigan, Ann Arbor, Michigan
}

\begin{abstract}
Aim: The intent of the present study was to evaluate changes in ventricular function with percutaneous closure of atrial septal defect (ASD), as it is associated with alterations in ventricular loading and function. Transcatheter occlusion of ASD imparts acute changes in volume loading of the left ventricle $(L V)$ that obscures measurement of ventricular function by load-dependent indices. To differentiate between changes in ventricular loading and function, load-independent indices of ventricular function must be utilized.

Methods: During transcatheter occlusion of ASD, subjects underwent measurement of LV pressure and volume by the conductance catheter method. Load-dependent indices of ventricular function included: systolic and diastolic pressures, $+d P / d t_{\max }$, and $-d P / d t_{\max }$. Load-independent indices included: elastance and tau, the preloadindependent time constant ofisovolumic relaxation. To obtain elastance, afterload was augmented by phenylephrine bolus pre- and post-device occlusion.

Results: In total, 29 patients (age 2-79 years) underwent ASD device occlusion (device size 12-38 mm, median $28 \mathrm{~mm}$ ). Load-dependent indices were obtained in all, and satisfactory pressure-volume loops in 11. At baseline, $L V$ end-diastolic pressure was $5-23 \mathrm{mmHg}(13 \pm 5 \mathrm{mmHg})$ and tau was $31 \pm 6 \mathrm{~ms}$. Postclosure of the ASD, LV systolic and diastolic pressures rose by $10 \pm 11 \mathrm{mmHg}$ and $5 \pm 3 \mathrm{mmHg}$, respectively $(P<0.05)$, and $+d P / d t_{\max }$ rose from $1,288 \pm 313 \mathrm{mmHg} / \mathrm{sec}$ to $1,415 \pm 465 \mathrm{mmHg} / \mathrm{sec}(P<0.05)$, but $-d P / \mathrm{dt}_{\max }$ was unchanged. Elastance significantly improved $(9.4 \pm 8.3 \mathrm{mmHg} / \mathrm{mL}$ vs. $13.0 \pm 7.3 \mathrm{mmHg} / \mathrm{mL}, P<0.05)$ and tau was unchanged.

Conclusions: Transcatheter occlusion of ASD is associated with acute improvement in load-independent indices of systolic function in this cohort, without significant worsening of the preload-independent index of diastolic function. (J Interven Cardiol 2014;27:204-211)
\end{abstract}

\section{Introduction}

Previous work has shown that patients with an atrial septal defect (ASD) and right ventricular volume overload have impaired left ventricular systolic function at baseline, and an impaired functional reserve. ${ }^{1-3}$ At time of either surgical or transcatheter closure, there is significant effect on cardiac mechanics caused by left ventricular volume loading, right ventricular volume unloading, and structural changes in the atrial septum. ${ }^{4}$ Along with the effect of these

Address for reprints: D. S. Lim, M.D., Heart \& Vascular Center, University of Virginia, Hospital Expansion, 4th Floor, Room 4038, 1215 Lee Street, Charlottesville, Virginia 22908-0386. Fax: 434982-6139; e-mail: SL9PC@virginia.edu loading and structural changes, there are reports of impairment in ventricular function after surgical ASD closure, both in the short term, as related to cardiopulmonary bypass, and in the longer term. ${ }^{5-8}$ Additionally, there have been reports in older patients with ASD of acute left ventricular failure after surgical repair of the ASD. ${ }^{9,10}$ While transcatheter approaches avoid the detrimental effect of cardiopulmonary bypass, concern has arisen about the potential of the device, being more rigid than the surrounding tissue, to create altered atrial and ventricular mechanics. ${ }^{11}$ Previously, detailed analysis of the effect of percutaneous device ASD closure has been mainly limited to load-dependent indices. ${ }^{5-8,11}$ However, in order to assess the effect on ventricular function with the closure of large atrial shunts, preload-independent indices must be utilized. 


\section{Materials and Methods}

This study was approved by the Institutional Review Board/Human Investigations Committee at the University of Virginia, and all patients gave informed written consent prior to study enrollment.

Study inclusion criteria included patients with large ASD undergoing percutaneous transcatheter device closure, which required arterial access for the procedure. Patients with additional congenital cardiac lesions were excluded.

All studies were performed in patients under general anesthesia, with breath-hold during measurements. Study measurements were performed both prior to and within 5 minutes after device ASD occlusion. Standard right and left heart hemodynamic measurements were obtained using fluid-filled catheters. High-fidelity left ventricular pressure measurements were obtained using 3- or 5-French Millar Mikro-Tip Multi-Segment Pressure-Volume catheters (SSD-1013 and SPC-550 catheters, Millar Instruments, Houston, TX). Realtime left ventricular volumetric measurements were obtained by connecting the left ventricular catheter to a CFL-512 Cardiac Function Computer (CD Leycom, Zoetermeer, The Netherlands). ${ }^{12,13}$ Consistent conductance catheter position was confirmed by both fluoroscopy (Fig. 1) and by localizing the superiormost volume segment(s) in the aorta, which was confirmed by a clockwise pressure-volume loop (Fig. 2). A clockwise pressure-volume loop was defined as rise in both the chamber pressure and volume that occurs in the aorta but not in the ventricle. The conductance catheter position was maintained throughout the procedure, and the sensing segments were kept the same both prior to and after the ASD closure. Real-time pressure-volume loops were obtained after correction for blood resistivity (rho). Parallel conductance $(\mathrm{Vc})$ correction was performed by injecting $10 \mathrm{~mL}$ of hypertonic (3\%) saline in a pulmonary artery catheter, as per the method of dilution. ${ }^{14}$ As each patient was to serve as his own control, $\alpha$ correction for absolute volume measurements was not performed. By augmentation of afterload with phenylephrine bolus, the end-systolic pressure-volume relationship (elastance) was obtained (Fig. 3).

Load-dependent indices of ventricular function included high-fidelity pressure measurements, which were plotted with respect to time, as was the first derivative $(\mathrm{dP} / \mathrm{dt}) .+\mathrm{dP} / \mathrm{dt}_{\max }$ and $-\mathrm{dP} / \mathrm{dt}_{\max }$ were then

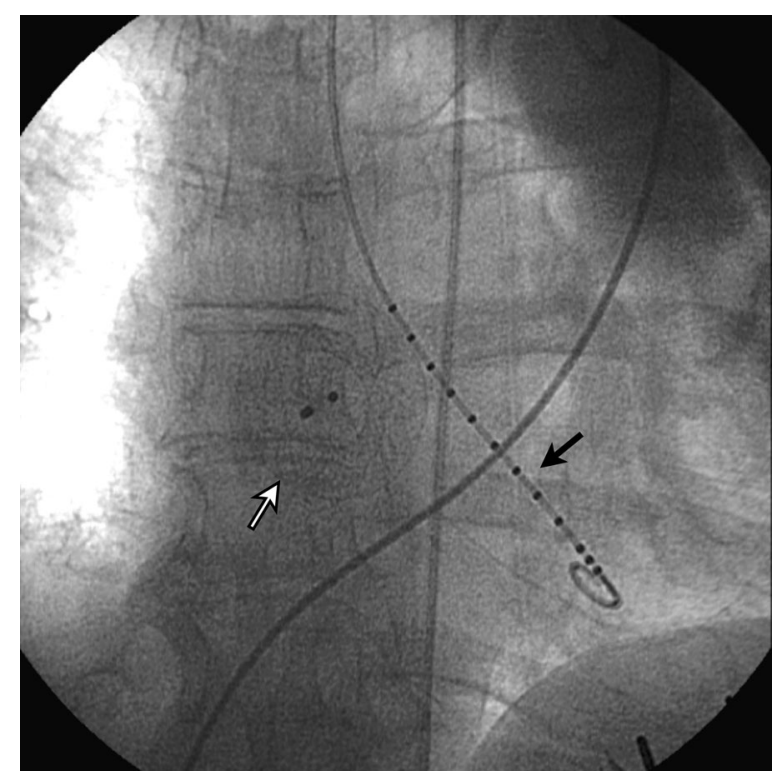

Figure 1. Cineradiogram of retrograde placement of conductance catheter in LV. The closed black arrow indicates the conductance catheter positioned through a retrograde approach into the body of the LV. Stable position during measurements is confirmed both by loop analysis and by fluoroscopic markers.

taken as the maximum and minimum of averaged values for three consecutive beats at steady state, respectively (Fig. 4). Tau, the preload-independent time constant of isovolumic relaxation, was the time for ventricular pressure to fall from $-\mathrm{dP} / \mathrm{dt}_{\max }$ to the inverse natural $\log$ of that pressure. ${ }^{15}$ Since tau is quite sensitive to changes in afterload, we measured it at baseline prior to augmentation of afterload, and again after ASD closure, and again prior to augmentation of afterload. The preload-independent index of ventricular systolic function measured was the elastance or the end-systolic pressure-volume relationship. ${ }^{16-18}$ End systole was defined as the point on the pressure-volume loop where the pressure/volume ratio was at maximum. With augmentation of afterload by phenylephrine bolus, a series of pressure-volume loops was obtained (Fig. 3), and the slope of the end-systolic points was plotted, with the slope defining elastance. ${ }^{17}$ Left ventricular end-diastolic pressure was measured on the pressure tracing coincident with the electrocardiographic R wave.

Patients ASD were then closed using the Am-platzer Septal Occluder (AGA Medical, Golden Valley, MN), as previously described. ${ }^{19}$ Repeat hemodynamic and conductance catheter measurements were then obtained. 


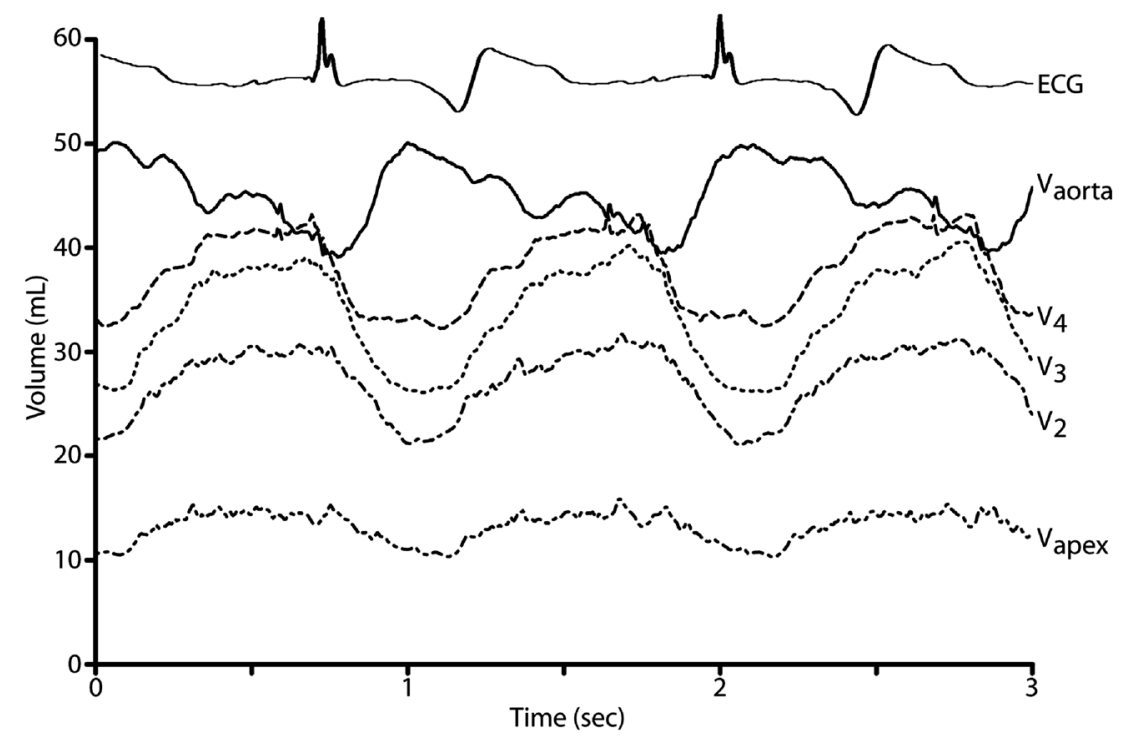

Figure 2. Catheter positioning by volume measurements. The first four volume segments, from $V_{x}$ to $V 4$, are located within the ventricle as demonstrated by the simultaneous decrease in volumes during systole and increase during diastole. The most superior segment, labeled $V_{\text {aorta }}$, is showing the expected volume rise as the aorta distends with systolic ventricular contraction, and the reverse during diastole.

Data were prospectively excluded for new-onset atrial dysrhythmias, catheter malpositioning, significant electrocardiographic (ECG) artifact, and unsuccessful device implantation.

Statistical Analysis. Each patient served as his own control, with pre- and post-ASD occlusion measurements compared by paired $t$-tests, with statistical significance defined as $\mathrm{P}<0.05$.

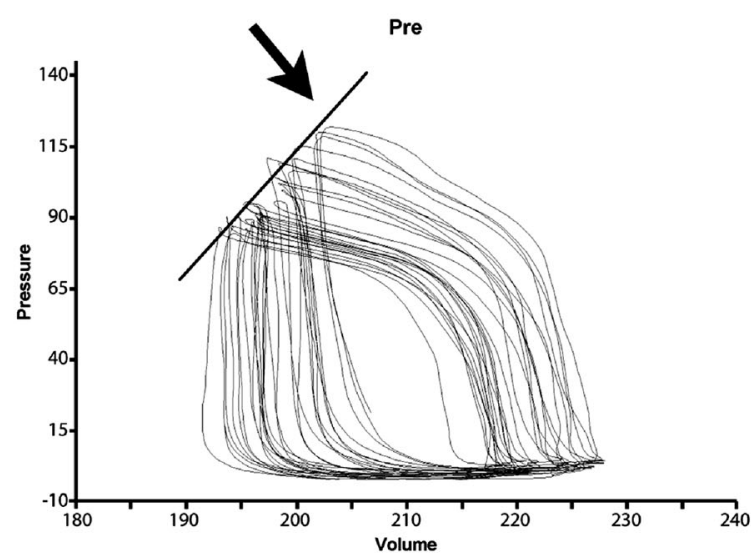

\section{Results}

Twenty-nine patients, with ages ranging from 2 to 79 years $(31 \pm 24$ years, see Fig. 5), with known large ASD and significant right ventricular dilatation, consented and enrolled in the study. Of the 29 study subjects, 27 had successful device implantation, and had study measurements performed both pre- and post-

Figure 3. Elastance pre- and postintervention. From a stable pressure-volume loop recording, loading conditions are changed with phenylephrine bolus (to augment afterload), and the series of end-systolic points is plotted on the graph on the left. The slope of this line (closed black arrow) yielded the value of elastance. After percutaneous device closure of the ASD was performed, elastance was remeasured and plotted on the graph on the right. 

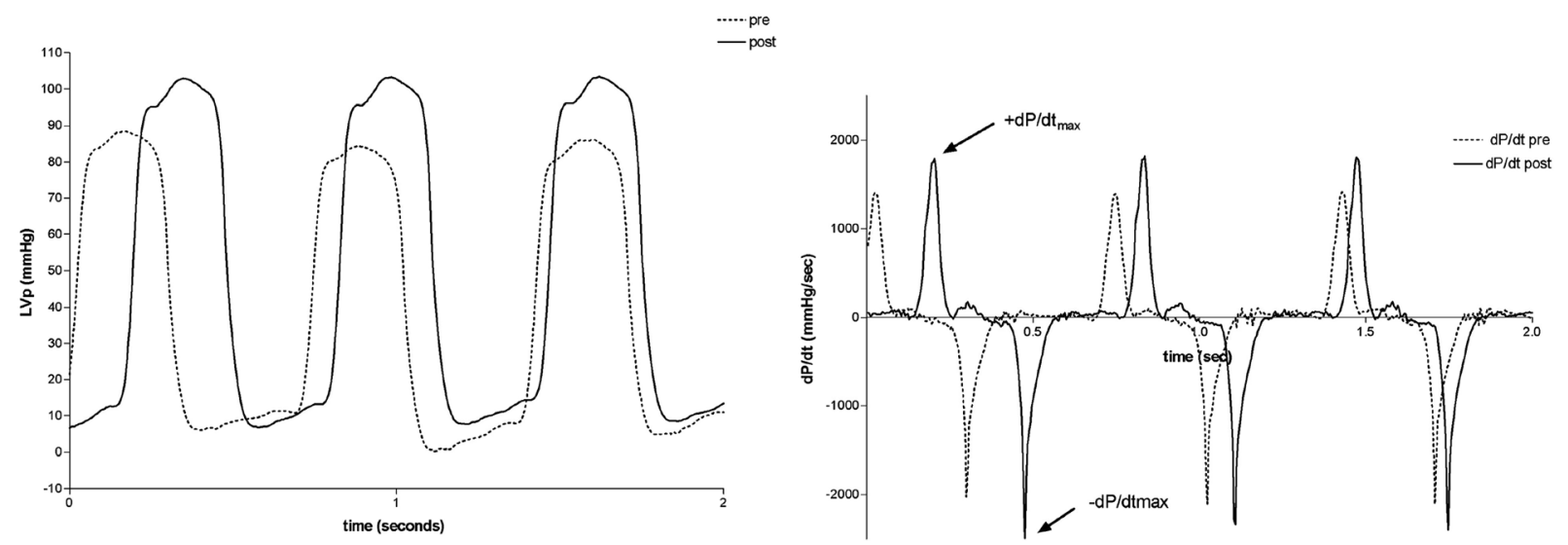

Figure 4. Change in LV pressure and first derivative. Changes in left ventricular pressure and the first derivative (dP/dt) are plotted with respect to time in the same patient, before and after ASD closure. Note the rise in both systolic and end-diastolic pressures with left ventricular volume loading, and the increase in positive and negative $\mathrm{dP} / \mathrm{dt}_{\max }$ (arrows).

device deployment. Of the two patients not receiving ASD devices, one was due to a stretch diameter of $>38 \mathrm{~mm}$, and the other was due to inadequate posterior rim. Of those receiving ASD device occlusion, the device sizes ranged from 12 to $38 \mathrm{~mm}$, with a median of $28 \mathrm{~mm}$, and left-to-right shunt ratio (Qp:Qs) measured under anesthesia was $2.5 \pm 0.8$ (range 1.54.1). At baseline, pulmonary artery systolic pressures were $32.9 \pm 13.7 \mathrm{mmHg}$ (range $17-75 \mathrm{mmHg}$ ), and left ventricular end-diastolic pressures were $13.1 \pm$ $4.7 \mathrm{mmHg}$ (range $5-23 \mathrm{mmHg}$ ).

Of the 27 subjects with successful ASD closure, load-dependent indices were obtained in all, and satisfactory pressure-volume loops in 11. Pressurevolume loops were excluded in 3 patients for acute development of atrial dysrhythmia, in 10 patients for

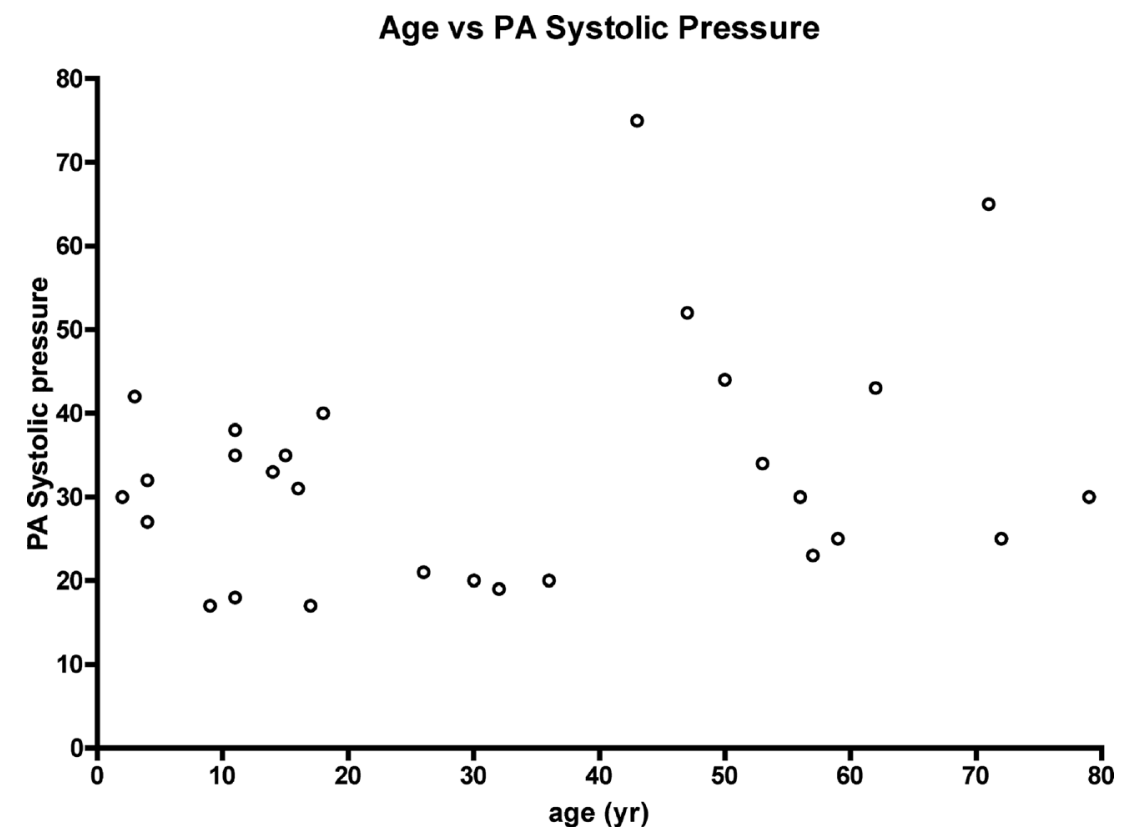

Figure 5. Age of patient cohort versus pulmonary pressure. The age of the patients at time of ASD closure is compared with pulmonary artery systolic pressure prior to ASD closure, as measured by catheterization. 
catheter malpositioning with phenylephrine bolus, which was determined by subsequent review of the segmental pressure-volume loops, (Fig. 2), and in 4 patients for significant ECG artifact that occurred when the walls of the ventricle contacted the conductance catheter.

There was no significant change in heart rate preversus post-ASD device deployment. After ASD device deployment, the pulmonary artery pressure fell by $4.0 \pm 8.2 \mathrm{mmHg}$ (range $8-33 \mathrm{mmHg}, \mathrm{P}<0.05$ compared with baseline). Left ventricular systolic pressure rose by $10 \pm 11 \mathrm{mmHg}$ (range -6-37 $\mathrm{mmHg}, \mathrm{P}<0.05$ compared with baseline), as did diastolic pressure by $5 \pm 3 \mathrm{mmHg}$ (range $0-12 \mathrm{mmHg}$, $\mathrm{P}<0.05$ compared with baseline). The $+\mathrm{dP} / \mathrm{dt}_{\max }$ rose from $1,265 \pm 322 \mathrm{mmHg} / \mathrm{sec}$ to $1,415 \pm 465 \mathrm{mmHg} / \mathrm{sec}$ by $7.1 \pm 23.4 \%$ (range $-50-60 \%, \mathrm{P}<0.05$ compared with baseline), but there was no significant change in $-\mathrm{dP} / \mathrm{dt}_{\max }$.

In the study subjects with adequate pressure-volume loop recordings pre- and post-ASD closure, elastance at baseline was $9.4 \pm 8.3 \mathrm{mmHg} / \mathrm{mL}$ (range $2-32 \mathrm{mmHg}$ / $\mathrm{mL}$ ). Notably, elastance rose in all but the eldest patient, in whom there was a marked drop (Fig. 6), and after ASD closure, it was $13.0 \pm 7.3 \mathrm{mmHg} / \mathrm{mL}$ (range 4-26 mmHg/mL, $\mathrm{P}<0.05$ compared with baseline, excluding eldest patient).

Tau, the preload-independent time constant of isovolumic relaxation, which was $30.9 \pm 6.5 \mathrm{~ms}$ (range
17-44 ms), showed no significant change with ASD closure (Fig. 7).

\section{Discussion}

In this cohort of patients with large ASD and right ventricular volume overload, percutaneous device closure of the atrial shunt reduced the pulmonary artery pressure, and raised both the left ventricular systolic and end-diastolic pressures. These findings are consistent with the sudden volume unloading of the pulmonary bed and concomitant volume loading of the left ventricle (LV). While this sudden increase in left ventricular preload should improve load-dependent indices of ventricular function, such as the commonly used ejection fraction, surgical series have shown an acute decrease in such indices, ${ }^{7}$ which is likely due to the deleterious effects of cardiopulmonary bypass. ${ }^{20}$ However, in this cohort undergoing percutaneous device ASD closure elastance, the preload-independent index of systolic ventricular function demonstrated improvement in all patients except the eldest. It is probable that the left ventricular geometric changes associated with volume unloading of the right ventricle allow improvement in left ventricular myocardial fiber arrangement that creates more favorable myocardial mechanics. While the concept of ventricular interdependence has been known for nearly 100 years, the

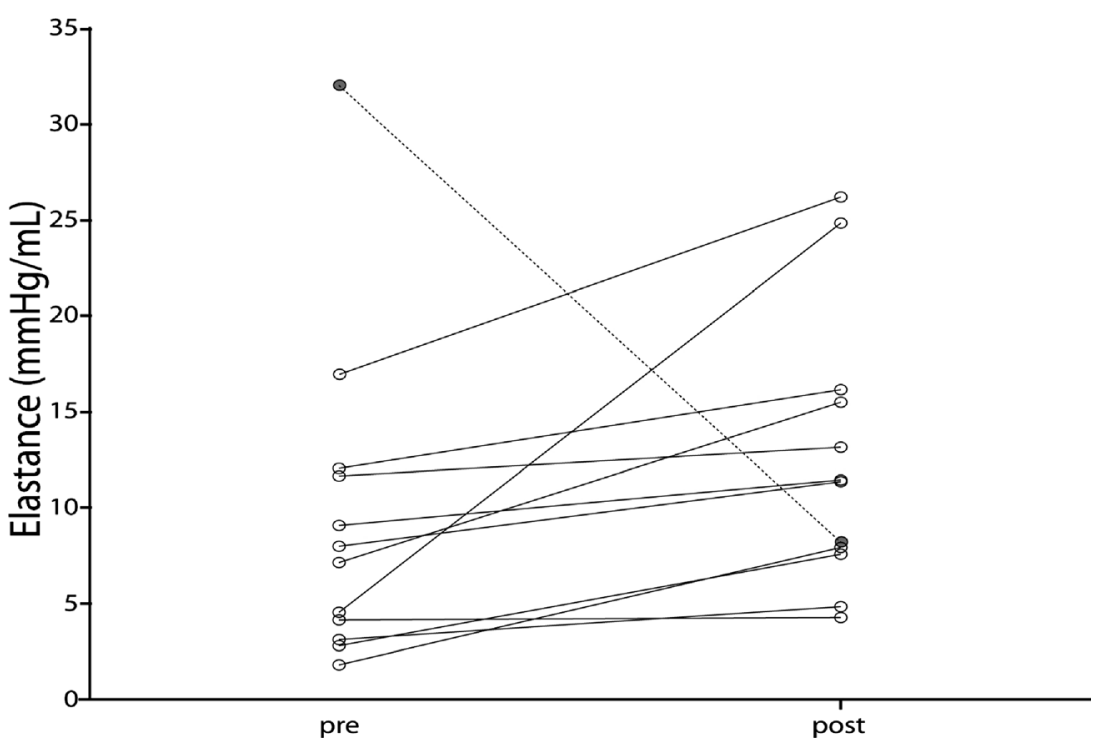

Figure 6. Change in left ventricular elastance. Elastance, a load-independent index of ventricular systolic function, is demonstrated in all patients who had satisfactory pressure-volume loops. 


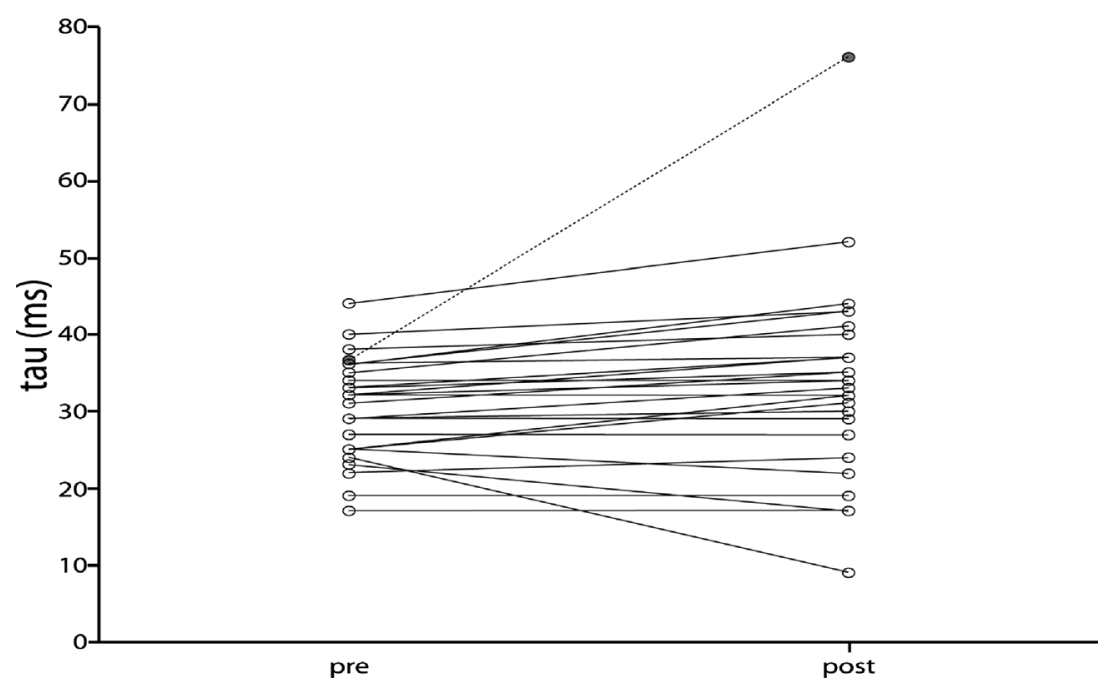

Figure 7. Change in left ventricular tau. Tau, a preload-independent index of ventricular diastolic function, was unchanged in the cohort measured.

nature of this mechanical coupling has not been completely determined. ${ }^{21}$ While pressure changes in one ventricle may affect contralateral ventricular performance, ${ }^{22}$ this present study looks specifically at changes in volume loading conditions as imparted by the ASD closure, which is a diastolic phenomenon. In canine studies, acute volume changes in the right ventricle have been shown to have a significant impact on left ventricular mechanics in the setting of an intact pericardium and significant right ventricular volume. ${ }^{23}$ We selected our patient cohort to have this same condition, and found that in most the change in right ventricular volume loading did benefit the left ventricular mechanics. However, it is also likely that older patients are less likely to have acute improvement in ventricular mechanics as their increasingly stiff ventricles may limit these geometric changes. It is interesting to note that in this patient cohort, despite the ages ranging into the 7th decade with significant ASD shunts and presumably stiff ventricles, there were no patients who had significant adverse sequelae after ASD closure.

The rise in left ventricular end-diastolic pressure seen in closure of the large atrial shunt in this cohort should not be interpreted as diastolic dysfunction related to the strain in handling the acute volume load. ${ }^{24}$ The absolute pressure measurement is clearly affected by acute change in loading conditions, as is the first derivative, $\mathrm{dP} / \mathrm{dt}_{\max }{ }^{25} \mathrm{Tau}$, which has been shown to be an index of diastolic function that is preload insensitive, ${ }^{15}$ showed no adverse effect of ASD device closure. Similarly, in canine models, the pericardium has been found to be the limiting factor in changes in diastolic function related to right and left ventricular loading changes. ${ }^{26}$ However, there may be an agerelated limitation imparted by the pericardium or the myocardium to the mechanical benefit of volume unloading in the right ventricle.

Another load-independent index of diastolic function is the end-diastolic pressure-volume relationship or compliance. Ideally, it should have been measured along with tau in this study. However, we found that as a limitation of the current conductance catheter technique, a prominent electrical artifact on the measured pressure-volume loop occurs at end diastole that we believe is due to the catheter oversensing ventricular depolarization. Unfortunately, this variable electrical artifact obscures the measurement of end diastole on the pressure-volume loop, and makes determination of compliance unreliable by this method. It is possible that future hybrid methods of pressure and volume measurements, combining invasive micromanometer pressure measurements with magnetic resonance imaging to determine changing volumes, will allow compliance to be reliably measured. ${ }^{27}$

Another technical issue involves the measurement of elastance that previously involved preload augmentation by temporary caval occlusion. ${ }^{13}$ However, we observed in our initial attempts in this patient population that changes in preload had a limited effect in creating the shift in the end-systolic pressure-volume point, and we were unable to determine the slope of 
those points. This was likely due to the presence of the large atrial shunt that by shunting the volume load from left to right limited the ability of preload occlusion to generate a range of left ventricular filling conditions for adequate elastance measurement. Since closing the shunt would change this effect and potentially confound measurements, we chose to determine elastance by afterload augmentation with phenylephrine bolus.

The conductance catheter technique makes assumptions regarding the left ventricular volume in that there is assumed to be circular symmetry around the long axis of the catheter. However, in the setting of significant volume overload of the right ventricle, the ventricular septum has flattened or paradoxical movement in diastole, and this, therefore, impacts this assumption. This present study was limited in that we did not perform simultaneous ventriculography before and after ASD closure to correlate the accuracy of absolute volume measurements of the LV with another standard, such as angiography. However, the values of the results described are expressed as changes in each patient, and the absolute value of elastance is not as important as the direction of its change with ASD closure. Additionally, that elastance improved in this cohort of human subjects mirrors the data from closed chest canine models of right ventricular volume overload. ${ }^{26}$

This study was limited by the reduced number of subjects' data available for pressure-volume loop analysis, which is due to the fickle nature of the conductance catheter technique. We frequently found difficulty in maintaining consistent catheter position in the ventricle with bolus dose of alpha agonists, which led to hyperdynamic ventricular contraction. Therefore, we developed strict criteria using both the fluoroscopic markers of catheter position, as well as aortic volume measurements from the conductance catheter, to ensure accurate volume measurements by this technique (Figs. 1 and 2).

Previously, surgical ASD closure has been associated with both acute and chronic deleterious effects on left ventricular function. ${ }^{5-7}$ These adverse effects of the surgical approach are on top of the LV with some degree of baseline dysfunction. ${ }^{1-3}$ While the long-term outlook after surgical ASD closure is generally thought to be excellent, there has been a widespread shift to percutaneous ASD closure. Fortunately, it appears that in this study's cohort of patients, percutaneous ASD closure was not associated with a significant decrease in either systolic or diastolic left ventricular function. This may be related to the deleterious effects on both ventricular function and systemic vascular resistance of cardiopulmonary bypass that is required for surgical closure. $^{20}$

\section{Conclusion}

In this cohort of patients with large atrial shunts, percutaneous device closure was associated with improvement in left ventricular systolic function, without worsening of diastolic function.

\section{References}

1. Flamm MD, Cohn KE, Hancock EW. Ventricular function in atrial septal defect. Am J Med 1970;48:286-294.

2. Walker RE, Moran AM, Gauvreau K, et al. Evidence of adverse ventricular interdependence in patients with atrial septal defects. Am J Cardiol 2004;93:1374-1377, A1376.

3. Popio KA, Gorlin R, Teichholz LE, et al. Abnormalities of left ventricular function and geometry in adults with an atrial septal defect. Ventriculographic, hemodynamic and echocardiographic studies. Am J Cardiol 1975;36:302-308.

4. Tanoue Y, Morita S, Ochiai Y, et al. Impact of atrial septal defect closure on right ventricular performance. Circ J 2006;70:909912.

5. Di Salvo G, Drago M, Pacileo G, et al. Comparison of strain rate imaging for quantitative evaluation of regional left and right ventricular function after surgical versus percutaneous closure of atrial septal defect. Am J Cardiol 2005;96:299-302.

6. Cheung YF, Lun KS, Chau AK. Doppler tissue imaging analysis of ventricular function after surgical and transcatheter closure of atrial septal defect. Am J Cardiol 2004;93:375-378.

7. Hanseus KC, Bjorkhem GE, Brodin LA, et al. Analysis of atrioventricular plane movements by Doppler tissue imaging and M-mode in children with atrial septal defects before and after surgical and device closure. Pediatr Cardiol 2002;23:152-159.

8. Dhillon R, Josen M, Henein M, et al. Transcatheter closure of atrial septal defect preserves right ventricular function. Heart 2002;87:461-465.

9. Beyer J. Atrial septal defect: Acute left heart failure after surgical closure. Ann Thorac Surg 1978;25:36-43.

10. Davies H, Oliver GC, Rappoport WJ, et al. Abnormal left heart function after operation for atrial septal defect. Br Heart J 1970;32:747-753.

11. Lange A, Coleman DM, Palka P, et al. Effect of catheter device closure of atrial septal defect on diastolic mitral annular motion. Am J Cardiol 2003;91:104-108.

12. Cassidy SC, Teitel DF. The conductance volume catheter technique for measurement of left ventricular volume in young piglets. Pediatr Res 1992;31:85-90.

13. Teitel DF, Klautz RJ, Cassidy SC, et al. The end-systolic pressure-volume relationship in young animals using the conductance technique. Eur Heart J 1992;13(Suppl E):40-46.

14. Baan J, van der Velde ET, de Bruin HG, et al. Continuous measurement of left ventricular volume in animals and humans by conductance catheter. Circulation 1984;70:812-823.

15. Gaasch WH, Blaustein AS, Andrias CW, et al. Myocardial relaxation. II. Hemodynamic determinants of rate of left ventricular isovolumic pressure decline. Am J Physiol 1980; 239:H1-H6. 


\section{EFFECT OF PERCUTANEOUS CLOSURE OF ASD ON VENTRICULAR FUNCTION}

16. Suga H, Sagawa K. Instantaneous pressure-volume relationships and their ratio in the excised, supported canine left ventricle. Circ Res 1974;35:117-126.

17. Suga H, Sagawa K, Shoukas AA. Load independence of the instantaneous pressure-volume ratio of the canine left ventricle and effects of epinephrine and heart rate on the ratio. Circ Res 1973;32:314-322.

18. Borow KM, Neumann A, Wynne J. Sensitivity of endsystolic pressure-dimension and pressure-volume relations to the inotropic state in humans. Circulation 1982;65:988-997.

19. Masura J, Gavora P, Formanek A, et al. Transcatheter closure of secundum atrial septal defects using the new self-centering Amplatzer septal occluder: Initial human experience. Cathet Cardiovasc Diagn 1997;42:388-393.

20. Wallace A, Lam HW, Nose PS, et al., Changes in systolic and diastolic ventricular function with cold cardioplegic arrest in man. The Multicenter Study of Perioperative Ischemia (McSPI) Research Group. J Card Surg. 1994;9:497-502.

21. Henderson Y, Prince AL. The relative systolic discharges of the right and left ventricles and their bearing on pulmonary congestion and depletion. Heart 1914;5:217-226.
22. Little WC, Badke FR, O'Rourke RA. Effect of right ventricular pressure on the end-diastolic left ventricular pressure-volume relationship before and after chronic right ventricular pressure overload in dogs without pericardia. Circ Res 1984;54:719730.

23. Maughan WL, Sunagawa K, Sagawa K. Ventricular systolic interdependence: Volume elastance model in isolated canine hearts. Am J Physiol 1987;253:H1381-H1390.

24. Diamond G, Forrester JS, Hargis J, et al. Diastolic pressurevolume relationship in the canine left ventricle. Circ Res 1971;29:267-275.

25. Cohn PF, Liedtke AJ, Serur J, et al. Maximal rate of pressure fall (peak negative $\mathrm{dP}-\mathrm{dt}$ ) during ventricular relaxation. Cardiovasc Res 1972;6:263-267.

26. Maughan WL, Kallman CH, Shoukas A. The effect of right ventricular filling on the pressure-volume relationship of ejecting canine left ventricle. Circ Res 1981;49:382-388.

27. Kuehne T, Yilmaz S, Steendijk P, et al. Magnetic resonance imaging analysis of right ventricular pressure-volume loops: In vivo validation and clinical application in patients with pulmonary hypertension. Circulation 2004;110:2010-2016. 